\title{
Predictive value of fluorodeoxyglucose uptake in head and neck cancer: importance of standardization
}

\author{
Editorial on EAORL-D-10-00277
}

\author{
Remco de Bree • Otto S. Hoekstra
}

Received: 2 July 2010/Accepted: 10 July 2010/Published online: 4 August 2010

(C) Springer-Verlag 2010

In the past the majority of patients with advanced stage head and neck squamous cell carcinoma (HNSCC) were treated with a combination of surgery and radiotherapy, often at the expense of functional and cosmetic morbidity, and thus reduced quality of life. Nowadays, in an attempt to decrease the morbidity, non-surgical treatments for advanced HNSCC are increasingly applied with considerable complete remission rates. It appeared that new radiation schemes (accelerated or hyperfractionated) and combinations of chemotherapy and radiotherapy all increase remission rates. When starting an organ sparing non-surgical therapy for functionally inoperable HNSCC (i.e. too high morbidity of surgical treatment expected), salvage surgery is held in reserve for residual or recurrent disease. However, salvage surgery with curative intent is only possible in about half of such patients. Moreover, the complication rate of salvage surgery after chemoradiation is high, with wound healing problems as a well-known complication. Since non-surgical treatment itself is also associated with acute and long-term side effects, leading to compromised quality of life, patients undergoing primary non-surgical treatment (radiotherapy with or without chemotherapy) followed by salvage surgery are exposed to cumulative morbidity of all treatment modalities. Another important disadvantage of surgery for residual or recurrent

R. de Bree $(\bowtie)$

Department of Otolaryngology/Head and Neck Surgery,

VU University Medical Center, De Boelelaan 1117,

1081 HV Amsterdam, PO Box 7057,

1007 MB Amsterdam, The Netherlands

e-mail: r.bree@vumc.nl

O. S. Hoekstra

Department of Nuclear Medicine \& PET Research,

VU University Medical Center, Amsterdam, The Netherlands tumour after radiotherapy is the fact that although it may be indicated postoperative radiotherapy is mostly no longer possible, limiting the oncological outcome of this treatment.

Because salvage treatment after (chemo)radiation carries a questionable prognosis but a high incidence of complications, (chemo)radiation may not be the choice of treatment in all patients with advanced HNSCC. A reliable predictor of outcome after chemoradiation is needed to select patients who are likely to benefit from non-surgical treatment.

Valuable predictive factors provide information on the outcome of therapy in an individual patient allowing avoidance of over- as well as undertreatment. In the present context, better selection may refrain a substantial number of patients from futile extensive and toxic treatment (radiation with or without chemotherapy), decrease the complication rate of surgical treatment and reserve radiotherapy for a postoperative setting if indicated.

Conventional prognostic factors for locoregional control include $\mathrm{T}$-stage, $\mathrm{N}$-stage and tumour site, grade and volume. The predictive value of molecular biological markers is currently under investigation. Tumour metabolism is another potential prognostic factor and can be studied with positron emission tomography (PET). Up-regulation of glucose uptake through overexpression of glucose transporters is an early event in malignant transformation. 18Fluoro-2-deoxyglucose (FDG) is the most widely used PET tracer in oncologic PET studies and can be used to measure the glucose metabolism in malignant tissues. Quantification of tracer uptake can be done in several ways, from pure visual analysis to simple calculations of uptake level using a Standardized Uptake Value (SUV; ie. the ratio of measured activity in a static scan obtained e.g. 60 min after FDG injection over the injected dose and 
normalized for volume of distribution, e.g. body weight) and to the standard PET approach of the more complicated kinetic analyses providing the metabolic rate of glucose uptake (in $\mu \mathrm{mol} / \mathrm{min} / \mathrm{g}$ ). There is a good reproducibility of metabolic measurements in malignant tumours using FDGPET [1]. In untreated primary HNSCC a high correlation was found between kinetic modelling and simple calculations with SUV [2]. There is ample proof of principle that quantitative PET measures have clinical value beyond visual interpretation. This pertains to prognostic (typically inverse relation between FDG uptake level and outcome) as well as predictive aspects (response monitoring). However, meta-analysis of such data is difficult because SUV proved anything but standardized in clinical practice. SUV results highly depend on several aspects that need to be controlled. This has now been recognized and the procedures to achieve standardised results are now in place [3]. Using these guidelines, larger multicentre studies and meta-analyses are possible to define clinically appropriate, externally validated thresholds and criteria.

In HNSCC, SUV during treatment appeared to be more reliable than visual assessment in predicting treatment outcome [4]. High SUV pretreatment predicts for significantly worse outcome [5-7]. FDG uptake, as measured by SUV, prior to radiotherapy with or without chemotherapy for stage II-IV HNSCC has a potential value in predicting local control, with reported local control rates of 86 and $55 \%$ for low ( $\leq$ median) and high SUV, respectively [8]. Other studies reported similar effects on survival. Therefore, some authors concluded that patients with higher FDG uptake pretreatment should be considered for a more aggressive treatment approach or modification of (different) treatment modalities to acquire sufficient tumour control [9]. Sofar, in part due to the interinstitute lack of standardised PET methods, no reproducible numerical SUV threshold has been found [10]. Currently, no SUV cut-off value has been established to stratify patients pretreatment in prognostically different subgroups. In the future SUV might be used for individualized treatment planning. Inokuchi et al [11] found that pretreatment SUV of nodal disease was one of the strongest predictive factors and also provided important information for the selection of patients suitable for planned neck dissection after chemoradiation. Unfortunately, no correction for lymph node volume was performed: due to partial volume effects smaller lymph nodes will always have lower SUV. Therefore, the predictive value of uncorrected SUV may be highly depend on (conventional) size criteria.

In this issue of European Archives of Otorhinolaryngology Haerle et al. [12] examined the correlation between the pretreatment maximal SUV (SUVmax) and conventional prognostic factors. SUVmax appears to correlate with T-stage, but not with tumour grade and site. These findings do not preclude SUVmax from being a potential predictor. Although this would require a larger study, it may be more important to find prognostic value of SUVmax within groups of patients with conventional predictive factors, e.g. T-stage and subsite. Since smaller tumours suffer relatively more from partial volume effects (underestimating true FDG uptake), it might be expected that early T-stages have lower SUVmax values, as found in their study. The authors correctly conclude that the role of primary tumour SUV as a predictor of outcome or survival remains unclear and further larger studies are needed. Once the new PET-guidelines [3] have been implemented, this should become much less of a task than sofar.

Another approach is predicting the outcome in the early treatment phase, a clinically significant moment when switching to a surgical treatment is still possible, before the appearance of side-effects associated with irradiation and holding sufficient irradiation in reserve for postoperative adjuvant treatment. Since conventional CT and MRI can not reliable distinguish between tumour and postradiation changes, these anatomical imaging techniques are not useful in this approach. An effective therapy could very well result in early subclinical alterations in tumour physiology and biochemistry long before changes in tumour mass become apparent. Tumour metabolism during cytotoxic therapy can be studied within PET [13]. Several studies report on the association of FDG uptake in the early phase of treatment and final tumour response. In HNSCC, FDG-PET uptake during radiotherapy (after $47 \mathrm{~Gy}$ ) correlates with overall survival [4]. It is also shown that in the early phase of non-surgical treatment (radiotherapy with or without chemotherapy) FDG-uptake levels lower than the median value were associated with a significant higher local control rate compared to higher FDG-uptake: $96 \%$ and $55 \%(\mathrm{p}=0.002)$ respectively [2]. Unfortunately, this analysis did not account for effect-modification or confounding by stage

Whether metabolic changes predict clinical response is an evolving field of interest in oncology. Clinical studies in several types and sites of cancer report correlations between decline in tracer uptake and a positive outcome. If patients with an adenocarcinoma of the oesophageal junction were classified as metabolic responders when the metabolic activity (SUV) of the primary tumour on the FDG-PET 14 days after the start of neoadjuvant chemotherapy had decreased by more than $35 \%$ in comparison with the pretreatment FDG-PET, a predictive value for residual disease of $95 \%$ is found [14]. For gastric carcinoma this figure is $86 \%$ [15]. In HNSCC, SUV reduction predicts outcome after 2 or 3 cycles of chemotherapy [16]. Unfortunately, no cut-off level was established which can be used for eventual treatment modification. There are no such clinical studies on (chemo)radiation in HNSCC. 
FDG-uptake might have predictive value before and early during non-surgical treatment and may be used in future to individualize treatment planning. For HNSCC, only small studies without an established cut-off level are performed. Large multicentrer studies are needed to determine and validate the cut-off level and value of FDG uptake in predicting treatment outcome. PET quantification with SUVs is affected by many technical and physiological factors. As a result some of the variations in the literature on SUV-based patient outcomes are explained by differences in FDG-PET study methods [17]. These differences concern patient factors (e.g. plasma glucose levels), time interval between injection and scanning, image reconstruction technique, image quality and SUV measure used. Therefore, different studies are difficult to compare and meta-analysis will not be possible. In designing prospective multicenter studies to examine the value of SUV in the prediction of treatment outcome, calibration of PET-scanners and standardisation of PET-scanning protocols should be performed. Guidelines for standardisation and quantification of FDG uptake have been developed. A great challenge in the near future will be to implement and maintain standards in large multicenter trials [3]. Multicenter studies including large series of homogenous groups of patients with standardized FDG-PET scanning and quantification protocols are needed to determine the optimal cut-off level and clinical value of SUV in treatment planning of HNSCC patients.

\section{References}

1. Weber WA, Ziegler SI, Thödtmann R et al (1999) Reproducibility of metabolic measurements in malignant tumors using FDG PET. J Nucl Med 40:1771-1777

2. Brun E, Kjellen E, Tennvall J et al (2002) FDG PET studies during treatment: prediction of therapy outcome in head and neck squamous cell carcinoma. Head Neck 24:127-135

3. Boellaard R, O'Doherty MJ, Weber WA et al (2010) FDG PET and PET/CT: EANM procedure guidelines foor tumour PET imaging: version 1.0. Eur J Nucl Med Mol Imaging 37:181-200

4. Farrag A, Ceulemans G, Voordeckers M et al (2010) Can 18FFDG-PET response during radiotherapy be used as a predictive factor for the outcome of head and neck cancer patients? Nucl Med Commun 31:495-501

5. Minn H, Lapela M, Klemi PJ et al (1997) Prediction of survival with Fluorine-18-Fluoro-deoxyglucose and PET in head and neck cancer. J Nucl Med 38:1907-1911

6. Kim SY, Roh JL, Kim RA et al (2007) Use of 18F-FDG PET for primary treatment strategy in patients with squamous cell carcinoma of the oropharynx. J Nucl Med 48:752-757

7. Halfpenny W, Hain SF, Biassoni L et al (2002) FDG-PET. A possible prognostic factor in head and neck cancer. Br J Cancer 86:512-516

8. Allal AS, Dulguerov P, Allaoua M et al (2002) Standardized uptake value of 2-[18F] fluoro-2-deoxy-D-glucose in predicting outcome in head and neck carcinomas treated by radiotherapy with or without chemotherapy. J Clin Oncol 20:1398-1404

9. Torizuka T, Tanizaki Y, Kanno T et al (2009) Prognostic value of 18F-FDG PET in patients with head and neck squamous cell cancer. AJR Am J Roentgenol 192:W156-W160

10. Machtay M, Natwa M, Andrel J et al (2009) Pretreatment FDGPET standardized uptake value as a prognostic factor for outcome in head and neck cancer. Head Neck 31:195-201

11. Inokuchi $H$, Kodaira $T$, Tachibana $H$ et al (2010) Clinical usefulness of [(18)F] Fluoro-2-Deoxy-d-Glucose uptake in 178 headand-neck cancer patients with nodal metastasis treated with definitive chemoradiotherapy: consideration of its prognostic value and ability to provide guidance for optimal selection of patients for planned neck dissection. Int J Radiat Oncol Biol Phys 2010Apr 29. [Epub ahead of print]

12. Haerle SK, Huber GF, Hany TF et al (2010) Is there a correlation between ${ }^{18}$ F-FDG-PET standardized uptake value, T-classification, histological grading and the anatomic subsites in newly diagnosed squamous cell carcinoma of the head and neck? Eur Arch Otorhinolaryngol. doi:10.1007/s00405-010-1348-2

13. Price P, Jones T (1995) Can positron emission tomography (PET) be used to detect subclinical response to cancer therapy? The EC PET Oncology Concerted Action and the EORTC PET Study Group. Eur J Cancer 31A:1924-1927

14. Ott K, Weber WA, Lordick F et al (2006) Metabolic imaging predicts response, survival and recurrence in adenocarcinoma of the esophagogastric junction. J Clin Oncol 24:4692-4698

15. Ott K, Fink U, Becker K et al (2003) Prediction of response to preoperative chemotherapy in gastric carcinoma by metabolic imaging; results of a prospective trial. J Clin Oncol 24:46044610

16. Dalsaso TA, Lowe VJ, Dunphy FR et al (2000) FDG-PET and CT in evaluation of chemotherapy in advanced head and neck cancer. Clin Positron Imaging 3:1-5

17. Boellaard R (2009) Standards for PET image acquisition and quantitative data analysis. J Nucl Med 50:11s-20s 